\title{
El aprendizaje experiencial: una práctica de innovación que se afianza en la Universidad Nacional del Litoral
}

\section{Gustavo Menéndez}

Secretario de Extensión de la

Universidad Nacional del Litoral,

Argentina.

gmenendez@unl.edu.ar
Laura Tarabella

Secretaria Académica de la

Universidad Nacional del Litoral.

Itarabella@rectorado.unl.edu.ar
Integración de la docencia y la extensión /

Desafíos de gestión
RECEPCIÓN: 24/06/16

ACEPTACIÓN FINAL: 07/08/16

\section{Resumen}

El presente artículo tiene como referencia el trabajo realizado por la Universidad Nacional del Litoral en lo que hace a la integración de la extensión con la docencia en los últimos años con el objetivo de promover una formación integral para todos los estudiantes que cursan las carreras de grado de nuestra Universidad.

El punto de partida es el reconocimiento de la extensión universitaria como una de las funciones sustantivas de la Universidad. Se define un enfoque teóricoconceptual que permite una caracterización de la extensión en términos de múltiples dimensiones: institucional, comunicacional, social, política y pedagógica. A partir de esta última se propone incorporar las prácticas de extensión en el currículo universitario desde una perspectiva epistemológica, pues este vínculo produce conocimiento y es justamente sobre el cual se sustenta y se inscribe la educación experiencial para inclusión en el currículo universitario.

Esta propuesta constituye una importante innovación en materia curricular, en tanto resignifica las prácticas de enseñanza y promueve instancias complementarias y valiosas en la formación de los estudiantes, a la vez que permite consolidar la extensión como una de las funciones sustantivas de la Universidad.

\section{Palabras clave}

- Extensión universitaria

- Educación experiencial

- Innovación curricular

\section{Resumo}

Este artigo tem como referência o trabalho feito pela Uniersidade Nacional del Litoral a respeito da integração da extensão com a docência nos últimos anos com o objetivo de promover uma formação integral dos alunos que fazem os cursos de graduação de nossa universidade.

O ponto de partida é o reconhecimento da extensão universitária como uma das funções essenciais da Universidade. É definido um enfoque teórico-conceitual qu permite uma caracterização da extensã em termos de dimensões múltiplas: institucional, comunicacional, social, política e pedagógica. A partir desta última se propõe incluir as práticas de extensão no currículo universitário baseadas em uma perspectiva epistemológica, já que este vínculo produz conhecimento e é justamente nele que se sustenta e se inscreve a educação experiencial para inclusão no currículo universitário.

Este projeto constitui uma inovação importante no currículo, já que ressignifica as práticas de ensino e promover instâncias complementares e valiosas na formação dos estudantes, e também permite consolidar a extensão como uma das funções essenciais da Universidade.

Palavras-chave

- Extensão universitária

- Educação experiencial

- Inovação do currículo
Para citación de este artículo

Menéndez, G. y Tarabella, L. (2016). El aprendizaje experiencial: una práctica de innovación que se afianza en la Universidad Nacional del Litoral. En Revista +E versión digital, (6), pp. 96-103. Santa Fe, Argentina: Ediciones UNL. 


\section{Introducción}

Uno de los desafíos más importantes que hoy enfrentan las universidades nacionales está relacionado con el logro de una mayor institucionalización y reconocimiento académico de la extensión universitaria, en tanto constituye una de sus funciones sustantivas. Es decir, la extensión forma parte de la vida académica que, integrada con la investigación y la docencia, contribuye de manera significativa a una mejor calidad y pertinencia universitaria y participa en los procesos de enseñanza y aprendizaje, en la generación de nuevos conocimientos y en su apropiación social con el fin de concentrar esfuerzos para una mayor inclusión y cohesión social.

En este sentido, al momento de plantearse la búsqueda de un mayor grado de institucionalización de la extensión se requiere realizar un profundo recorrido institucional que parte de la definición de principios y valores acerca de la educación en general y de la universidad en particular, así como del enfoque teórico conceptual desde el cual se va a plantear la extensión universitaria. Además, se necesita que esta función esencial se encuentre incorporada institucionalmente no solo en el estatuto de cada universidad sino también en sus planes de desarrollo institucional, en sus políticas institucionales y en sus diferentes reglamentaciones. Luego, en este camino de consolidación de la inserción curricular de la extensión resulta fundamental que las políticas acordadas en la universidad en materia de extensión universitaria se traduzcan en sus diversos instrumentos de gestión que permitan identificar las asignaciones de recursos, la definición de las prácticas institucionales y la reflexión crítica de las mismas.

Éste es el recorrido institucional que ha seguido la extensión en el caso de la Universidad Nacional del Litoral (UNL). La extensión forma parte indisoluble de un modelo de universidad que considera a la educación como un bien público social y un derecho humano y universal. Universidad que nace al calor de la Reforma Universitaria de 1918 y en la que la extensión ha sido sinónimo permanente de compromiso social, de inclusión, de diálogo y de democratización de los conocimientos, incorporada ya de esta manera en el primer estatuto fundante de la UNL en 1919.

Así, a partir de una profunda concepción democrática, autónoma, crítica y creativa, la UNL asume este compromiso social y promueve a través de sus políticas institucionales la más amplia democratización del saber, del conocimiento y de la cultura en diálogo permanente con la sociedad, brinda su desarrollo académico, científico y tecnológico e interactúa con diferentes actores sociales.

A su vez, la extensión en la Universidad ha sido la puerta de entrada al compromiso social, que se traduce en acciones y territorios determinados, en trabajos conjuntos con actores concretos.

Toda acción de extensión implica llevar adelante una práctica mediante la cual se pretende aportar a la solución o transformación de situaciones-problemas detectados en el medio. En cada práctica de extensión (que puede ser un programa, un proyecto, un conjunto de acciones, una capacitación, entre otros) se ponen en diálogo conocimientos científicos con saberes y conocimientos presentes en los espacios sociales en donde se trabaja. Este encuentro de conocimientos y saberes y diálogos con el Estado y la sociedad posibilita a la Universidad un enriquecimiento y fortalecimiento académico y permite pensar y repensar sus políticas de crecimiento institucional. Desde esta perspectiva, la formulación de las políticas de extensión es parte de la construcción de una agenda compartida entre los actores sociales, el Estado y la comunidad universitaria con profundo sentido académico y de pertinencia social.

La Universidad, mediante la extensión, promueve la apropiación social del conocimiento y la democratización del capital social y cultural, aspectos claves para contribuir a la transformación social, integrándose de esta manera al medio social del cual se nutre y al que permanentemente contribuye, consciente y comprometida con las problemáticas sociales, culturales y productivas de la región en la que se encuentra inserta.

La conjugación de las dimensiones (que se citan más adelante) y el modelo de intervención de la Secretaría de Extensión, posibilitaron a partir de 2014, la identificación de 12 ejes estratégicos para el desarrollo de la extensión:

1) Institucionalización y reconocimiento académico de la extensión.

2) Integración de la extensión con la docencia.

3) Integración de la extensión con la investigación.

4) Acción territorial de la Extensión.

5) Análisis y aportes a las políticas públicas.

6) Consolidación del Sistema Integrado de Programas y Proyectos de Extensión.

7) Fortalecimiento de la política editorial.

8) Formación y capacitación en extensión universitaria.

9) Internacionalización de la extensión.

10) Consolidación de líneas estratégicas de comunicación.

11) Planeamiento y evaluación de la extensión.

12) Fortalecimiento de los equipos de trabajo, presupuesto $y$ gestión de financiamientos.

Si bien la UNL exhibe una larga trayectoria en materia de extensión universitaria, en las últimas décadas ésta ha pasado a formar parte plena de la vida académica e institucional de la Universidad, con reconocimiento estatutario, incorporada a los planes de desarrollo institucional y fortalecida por un conjunto de normativas e instrumentos de gestión que hacen factible llevar a la práctica los enfoques teóricos conceptuales y metodológicos planteados y definidos por la institución en esta materia. 


\section{La extensión desde múltiples dimensiones}

En la introducción del Plan de Desarrollo Institucional 2010-2019 "Hacia la Universidad del Centenario", se expresa que uno de los propósitos de la UNL es formar ciudadanos críticos, con sólida formación profesional, actitud emprendedora, competencias para un desempeño internacional y compromiso social para integrarse a una sociedad democrática. Este ideario promueve la generación y gestión de propuestas académicas innovadoras, dinámicas y flexibles, que posibiliten el aprendizaje en situaciones reales, lo que da cuenta del diálogo entre la Universidad y el entorno social. Es así que las Secretarías de Extensión y Académica han promovido la reflexión acerca de los paradigmas educativos existentes y la propuesta de repensar otros que profundicen el pensamiento crítico y el compromiso social de los futuros profesionales de esta Universidad. Se hizo necesario, entonces, sistematizar categorías de análisis para configurar el marco dentro del cual pensar la extensión y su vinculación con la docencia a partir de múltiples dimensiones: institucional, comunicacional, social, política y pedagógica. ${ }^{1}$ En el Cuadro 1 se sintetizan esas dimensiones.

Cuadro 1

\begin{tabular}{ll}
\hline Académico & Supone: \\
institucional & - Reconocer el aporte de la extensión a la construcción y a la \\
& apropiación social del conocimiento. \\
& - Generar dispositivos de gestión que apuesten al \\
& fortalecimiento de las instituciones democráticas y de las \\
políticas públicas.
\end{tabular}

Política En tanto que toda acción de extensión tiene por objetivo transformar una situación determinada. Esto implica: - Interpelar al Estado y a las políticas públicas.

- Construir agendas de trabajo sobre aspectos relevantes que hacen a la cuestión social.
3. La integración de la extensión en los currículos de la UNL A partir de la petición que realizaran los estudiantes al Consejo Superior, se institucionaliza en la UNL la incorporación de las prácticas de extensión en el proceso formativo de los estudiantes de las carreras de grado de esta Universidad en tanto éstas constituyan interacciones e intervenciones sociales vinculadas a la formación profesional. Justamente, a través de la vigencia de este marco normativo (Resolución 274/07), las Secretarías de Extensión y Académica sentaron las bases para profundizar la legitimación de esta política de innovación curricular en el ámbito de las diferentes Unidades Académicas, y es el área de Incorporación Curricular de la Extensión la responsable de llevar adelante el plan de acción vinculado al proceso de curricularización de la extensión.

Desde el año 2007, la UNL viene trabajando en la incorporación curricular de Prácticas de Extensión de Educación Experiencial (PEEE) en todas sus carreras.

Esta propuesta es una importante innovación en materia curricular en tanto resignifica las prácticas de enseñanza y promueve instancias complementarias y valiosas en la formación de los futuros profesionales que tienen como propósito promover aprendizajes auténticos a partir de los cuales

“los estudiantes construyen conocimientos 'estratégicos' o 'condicionales' que implican saber por qué, dónde, cuándo y cómo se utilizan esos conocimientos, elaborados, particularmente, en relación con situaciones, casos, problemas o proyectos tal y como se encuentran en la vida real".

(Camilloni; 2013:11)

Las PEEE son una estrategia innovadora que permite la integración de la docencia y la extensión. En términos de E. Litwin:

"Entendemos por innovación educativa toda planeación y puesta en práctica creada con el objeto de promover el mejoramiento institucional de las prácticas de la enseñanza y/o de sus resultados. Las innovaciones responden a los fines de la educación y se inscriben con sentido en los contextos sociales, políticos e históricos de la vida de las instituciones. Creación, promoción del cambio y mejora son conceptos asociados a las innovaciones... las innovaciones pueden inscribirse en el corazón del currículo, esto es desarrollar contenidos de una manera novedosa y eficaz". (2008:65)

El desafío que se presenta es el diseño de estrategias, dispositivos y el impulso de acciones que admitan la construcción y apropiación por parte de estudiantes y docentes de prácticas de extensión bajo este enfoque y que promuevan su fortalecimiento. 


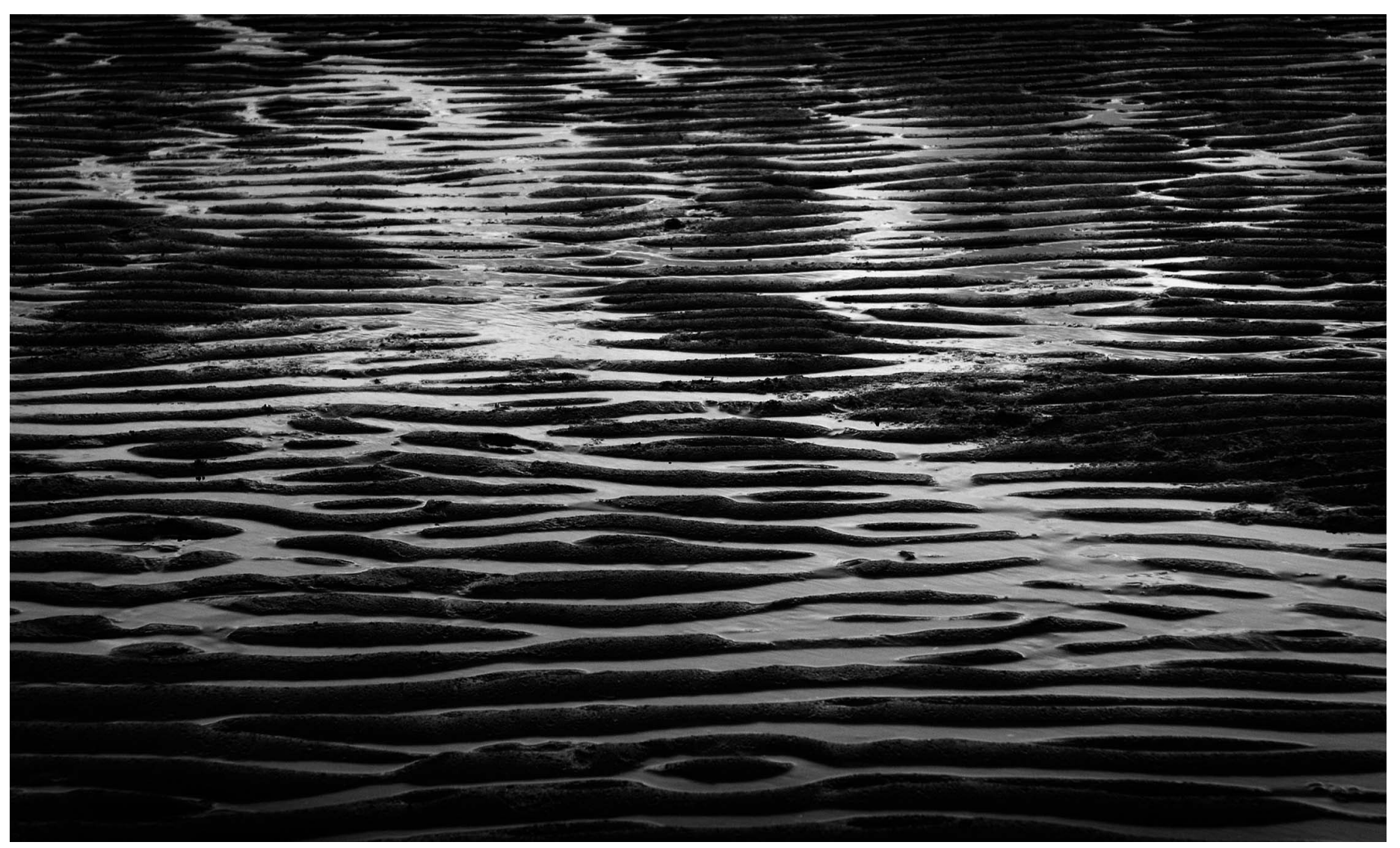

() Oscar Dechiara

El "Plan de Desarrollo Institucional", entre otras consideraciones relevantes, destaca que:

"la extensión es una función esencial de nuestra Universidad que promueve el diálogo permanente con el Estado - en sus diferentes jurisdicciones-, con otras instituciones educativas y científico tecnológicas del sistema provincial y nacional, con las organizaciones de la sociedad civil y de la producción en la búsqueda de soluciones a los problemas del contexto, lo que confiere a estas acciones una alta pertinencia social" y también donde se resalta que, "la integración entre la Extensión y la Enseñanza nos posiciona desde una perspectiva en la que los saberes culturales y los conocimientos académicos-científicos se ponen en juego con otros saberes y conocimientos que posee la sociedad, lo que posibilita analizar y abordar los problemas a partir de una concepción y comprensión de la complejidad". (20)
El Proyecto y Acción (PyA $)^{2}$ formulado en forma conjunta entre la Secretaría de Extensión y la Secretaría Académica recupera una larga tradición de esfuerzos por institucionalizar un nuevo paradigma en la manera de concebir la extensión desde una dimensión dialógica en tanto posiciona a la Universidad y a la sociedad en una relación compartida. Este diálogo supone también una perspectiva epistemológica, pues este vínculo produce conocimiento y es precisamente sobre el cual se sustenta y se inscribe la educación experiencial cuya inclusión en el currículo universitario "convierte a todos los que se impliquen seriamente en el proyecto curricular de formación, en interesados y protagonistas" (Camilloni, 2013:21). La gestión del PyA "Fortalecimiento de la Educación Experiencial en el currículo universitario" ha logrado reunir en las cinco convocatorias sucesivas, desde 2014, 97 proyectos de PEEE con sus respectivos docentes y equipos de cátedra responsables provenientes de todas las Unidades Académicas de la UNL (ver Listado 1).
2) Los Proyectos y Acciones (PyA) son las unidades operativas que materializan los objetivos del Plan de
Desarrollo Institucional 2010-2019. Son confeccionados por los diferentes actores participantes del proceso de planificación en colaboración con el equipo de la Secretaría de Planeamiento de la Universidad. 


\section{Propuestas de Prácticas de Extensión de Educación Experiencial 2014/2015/2016}

- Acompañamiento en la gestión de costos a emprendedores de la Economía Social y Solidaria en la ciudad de Santa Fe.

- Alimentación saludable vs. malnutrición.

- Análisis de la sustentabilidad ambiental de proyectos (optativa Facultad de Ingeniería y Ciencias Hídricas / electiva UNL)

- Aportes para reducir conflictos sociales relacionados a la falta de difusión y conocimiento de las nuevas prácticas de producción de frutillas en la zona periurbana de Coronda

- Aprender enseñando. La Química entre la universidad y la escuela secundaria. - Aprender Física haciendo Física. Propuesta y realización de trabajos prácticos de Física junto a estudiantes y docentes de la Escuela de Educación Técnica n 2025 Ceferino Namuncurá.

- Aprendiendo a cuidar nuestra salud, la de los demás y la del ambiente.

- Armado de un laboratorio de Química, capacitación y asesoramiento de los docentes para su uso e implementación en el ámbito de la enseñanza en una escuela primaria.

- Articulando la extensión en Medicina Veterinaria con políticas públicas en el Municipio de la ciudad de Esperanza.

- Asesoría pedagógica para la educación ambiental en el nivel primario: contribuciones desde la didáctica de la Geografía.

- Aspectos inherentes a la organización, administración y situación tributaria y laboral de las entidades deportivas en la actualidad. Aplicación en casos prácticos en territorio.

- Ayudar-t: aprendiendo sobre discapacidad para luego emprender la fabricación de productos de apoyo.

- Capacitación y gestión y administración para la comisión directiva de la Asociación Vecinal Villa del Parque.

- Caracterización de suelos para el mejoramiento de los cultivos y su rendimiento en una huerta escolar de la ciudad de Santa Fe.

- Cartografía temática isla La Rinconada - San Javier.

- Centro de Servicios en la Plaza de las Tres Culturas. Ciudad de Santa Fe,

República Argentina.

- Ciencia nómade: aprendiendo-enseñando.

- Ciudad intermedia: simulación (de un proceso) de planificación participativa para su ordenamiento territorial.

- Comportamiento social: conductas en conflicto con la salud y el aprendizaje.

- Conocer para mejorar la salud.
- Conocer y aprender los derechos desde una realidad auténtica para promover la construcción de ciudadanía.

- Conocer y aprender los derechos reales desde una dimensión dialógica y contextualizada en el sitio de producción conceptual.

- Conociendo desde el aprendizaje experiencial las practicas ganaderas que causan pérdidas económicas por decomiso de carne vacuna en la zona de influencia de la Facultad de Ciencias Veterinarias de la Universidad Nacional del Litoral.

- Construcción de un espacio de comercialización virtual para productores de la Economía Social y Solidaria en la ciudad de Santa Fe.

- Construyendo salud.

- Consultorios de la Facultad de Ciencias Jurídicas y Sociales / Asesoramiento Jurídico para la construcción de ciudadanía.

- Crianza artificial de terneros.

- Criterios lúdicos e informacionales para el diseño de dispositivos

comunicacionales: la promoción del concepto de soberanía alimentaria.

- Desarrollo de vínculos institucionales que promuevan experiencias de trabajo en producción agroecológica para estudiantes de agronomía en el Centro-Norte de la Provincia de Santa Fe.

- Descubriendo mecanismos de intervención comunitaria en seguridad alimentaria en una red institucional del barrio San Agustín.

- Detección temprana de problemáticas alimentarias y su abordaje en territorio.

- Detective de seres diminutos. Aprender jugando a prevenir las enfermedades parasitarias.

- Educación ambiental en la escuela primaria: asesoría pedagógica y construcción de propuestas didácticas innovadoras.

- Educación ambiental en las escuelas primarias de Esperanza, Santa Fe.

- Educación y derechos humanos en la primera infancia.

- Educando nuevos ciudadanos. La revalorización del patrimonio natural y cultural local: conociendo al Irupé.

- Educándonos como educadores en alimentación y nutrición.

- Ejercicio disciplinar para la construcción de ciudadanía.

- El árbol y sus aportes en los sectores periurbanos. "Construyendo un territorio para el futuro diferente".

- El mejoramiento urbano barrial como estrategia urbanística de inclusión social.

- Enemigos invisibles en mis alimentos. Estrategia didáctica aplicada a higiene

alimentaria y enfermedades transmisibles por alimentos.

Nota: las prácticas están ordenadas por orden alfabético. Algunas de ellas se reeditan año tras año. 
- Estrategias didácticas de nutrición en jardines maternales y educación inicial: el juego como propuesta de acción.

- Experiencia piloto para la construcción de un mapa de riesgo.

- Extensión universitaria: prácticas en el territorio.

- Formación de formadores de jóvenes de las escuelas medias en cambio climático.

- Formación universitaria y prevención de zoonosis parasitarias transmitidas por

los caninos: una experiencia en Esperanza.

- Fortalecimiento en la gestión de costos a emprendedores de la Economía Social y Solidaria de la ciudad de Santa Fe.

- Funciones del diseño de comunicación visual para el uso responsable del espacio público. Primera experiencia: Pista de Skate "Candioti Park".

- Inclusión de la tecnología satelital para la enseñanza de la Geografía: aprender y construir junto a otros sujetos en la escuela primaria.

- Inquietxs: derechos en movimiento. Construcción participativa de oportunidades ocupacionales para niñ@s y jóvenes.

- Jornadas Internacionales de Jóvenes Emprendedores. Programa de identidad y comunicación.

- Juego, ingenio y emoción: otra forma de aprender matemática.

- La biodiversidad de la reserva oeste de la ciudad de Santa Fe: un espacio para la ciencia ciudadana.

- La bioseguridad como herramienta de prevención y fomento de equipos de trabajo.

- La educación experiencial como herramienta de formación universitaria y prevención de zoonosis parasitarias transmitidas por los caninos.

- La implementación de estrategias urbanísticas de mejora barrial a partir del reconocimiento de lugares urbanos.

- La producción arquitectónica de la década de 1970 en la argentina. Una herramienta para la reflexión. Caso de Estudio: "Complejo habitacional San

Jerónimo Barrio Centenario - Santa Fe".

- Manejo del cultivo de arroz irrigado: visión técnica y social.

- Movilidad sostenible: caminos escolares.

- Nodo IV: Desarrollo Regional.

- Nutrición y estrategias didácticas aplicadas a los jardines maternales y educación inicial: El juego como propuesta de acción.

- Participando con ciencia.

- Planificación urbanística con sustentabilidad y participación. Un camino para

el ejercicio de la ciudadanía.
- Práctica hospitalaria de grandes animales.

- Práctica promocional de salud y derechos de Terapia Ocupacional.

- Prácticas académicas en espacios productivos vinculados con la biotecnología: aprendizaje experiencial en la cervecería Santa Fe.

- Problemática la relación existente entre la adolescencia y la imagen que los jóvenes tienen de sí mismos vinculante a los problemas existentes de índole alimentario, tales como la obesidad.

- Promoción de educación alimentaria en jóvenes y adultos: desafío conjunto Universidad-Comunidad.

- Promoviendo la formación profesional integral en medicina veterinaria desde el servicio social.

- Relaciones entre teorías sobre lengua y literatura y prácticas de enseñanza en espacios de formación de formadores (o la educación experiencial puesta en abismo).

- Relaciones que se construyen en las experiencias escolares. Condiciones socioafectivas y cognitivas que las posibilitan.

- Seguimiento epidemiológico de parasitosis en caninos del Barrio La Orilla, Esperanza, Santa Fe.

- Sistematización y análisis de las mediaciones de conflicto en Santa Fe.

- Social media en la empresa, herramientas de comunicación con el mercado.

- Taller de cerámica La Guardia: un aporte a la construcción de su identidad comunicacional.

- Trabajando juntos por una mejor producción en la empresa social caprina.

- Trabajo intergeneracional para mejorar la situación de salud de los adultos mayores.

- Un aporte tipográfico para la construcción colectiva de la identidad ciudadana.

- Un lugar para los guardianes del río: una oportunidad de materializar un habitar sustentable en el paisaje.

- Una intervención socioeducativa con alumnos del ciclo profesional de la carrera de Medicina Veterinaria.

- Una propuesta de aprendizaje experiencial en el Nuevo Hipódromo Las Flores, Santa Fe.

- Uso racional del equino de tiro en un barrio de la ciudad de Santa Fe.

- Vacunarnos es un derecho de mi familia y mi comunidad: ¡refuerza tu poder!

- Vigilancia Nutricional en escolares de la ciudad de Santa Fe.

Fuente: Área de Incorporación Curricular de la Extensión, Secretaría de Extensión UNL. 
Desde las primeras ideas plasmadas en el año 2007 a la configuración del actual escenario en el que las diez Unidades Académicas se han involucrado activamente en la propuesta, ha madurado la idea de que esta praxis — para cobrar relevancia e institucionalización - requirió de un tiempo y un proceso a través de los cuales se asumió la importancia de que, a los fines de superar una concepción reduccionista de la extensión, estos saberes fueran acreditados. Esto suponía necesariamente la curricularización de esta actividad, es decir, que la misma se pensara, diseñara y plasmara desde un espacio curricular. En este sentido, el documento "Experiencia Institucional. Documento General de Prácticas de Extensión en Educación Experiencial" de 2014 constituyó un punto de partida para la sistematización de las ideas y un insumo insoslayable para promover la formulación de los proyectos.

Para su presentación, los equipos de cátedra, asesorados por el Área de Incorporación Curricular de la Extensión, deben definir el objetivo de aprendizaje, la manera en que la Universidad puede contribuir al desarrollo local y/o regional considerando las políticas públicas vigentes, los criterios para la incorporación curricular y los modos de evaluación de los aprendizajes.

En suma, los proyectos responden a la idea de que los conocimientos se crean a partir de la transformación de la propia experiencia, esto es, las prácticas de extensión se integran al currículo y promueven que los estudiantes se involucren en la resolución de problemas reales en escenarios complejos. En muchos casos estas instancias son las primeras oportunidades de desarrollar experiencias de extensión y de fortalecer prácticas preprofesionales vinculadas al campo de formación. Estas propuestas requieren de una cuidadosa planificación con un alto grado de previsión y anticipación (Camilloni, 2013).

Desde esta perspectiva, la inclusión de la extensión en los currículos universitarios pretende la conversión de los conocimientos adquiridos en saberes o actitudes asociados a calificaciones académicas y profesionales.

\section{La formación en extensión}

Otro de los desafíos que se presenta es generar acciones continuas en la formación en aspectos pedagógicos de la Educación Experiencial de docentes y estudiantes. Esta formación sería un acompañamiento por parte de la Universidad a aquellos procesos que fueron suscitándose en el devenir de las prácticas. En palabras de Sandra Nicastro y María Beatriz Greco:

"el trabajo de acompañamiento hace un doble movimiento: mirar desde dentro de la situación y mirar de frente a la situación, como si uno invitara tomando de la mano al otro a salir de su escena y no enfrentarse a ella en el sentido de afrenta, sino de ponerse de cara a ella" (2012:99)
En tanto el objeto de acompañamiento es la problematización. Cuestión que, según las autoras:, "persigue como propósito provocar una nueva mirada sobre la situación que se atraviesa, hacer otras preguntas, establecer nuevas relaciones entre los hechos, las condiciones que los sostienen, etc." (citado por Nicastro y Greco en Nicastro y Andreozi. 2004:44).

Este acompañamiento que hace la institución a sus docentes y estudiantes fortalece y promueve, a partir de la participación en las PEEE, el compromiso de la comunidad educativa. Así, desde 2014 hasta la actualidad se han venido realizando talleres con referentes pedagógicos externos e internos en los que se registra un ascenso gradual de asistentes, docentes y estudiantes. Ascenso que evidencia una mayor cantidad de actores universitarios capacitados y formados bajo este enfoque de la Educación Experiencial y un aumento de los espacios curriculares que optan por esta manera de enseñar y de aprender.

Cabe resaltar que los estudiantes, más allá de los aprendizajes adquiridos a partir de la participación en las PEEE y de los talleres de capacitación realizados junto a los docentes, también tienen la oportunidad de formarse de manera teórica y práctica sobre extensión universitaria.

A partir de la Resolución 274/07 anteriormente nombrada, se efectuó una acción concreta desde el punto de vista académico: la creación de la asignatura electiva, que se viene desarrollando desde el año 2008, actualmente denominada: Extensión Universitaria: Prácticas en el territorio, que se ofrece a todos los estudiantes de grado de todas las carreras de la UNL. Esto ha posibilitado la formación integral de más de 500 estudiantes de esta casa de estudios.

Al hablar de todas las carreras de grado, se está diciendo también para todos los estudiantes interesados. Esta situación permite conformar un espacio curricular donde confluyen las distintas disciplinas y es factible abordar diversas problemáticas identificadas desde diversas perspectivas.

\section{Para seguir pensando...}

La búsqueda de estrategias didácticas posibilitadoras de la construcción y elaboración de nuevos aprendizajes llevará a plantear como alternativa posible la enseñanza a partir de las prácticas de extensión enmarcadas en la Educación Experiencial.

Se rescata también el trabajo interdisciplinario, que compromete no solo la cooperación entre las disciplinas presentes en la formación sino, esencialmente, la construcción colectiva y consensual del proyecto de formación, universidad-sociedad, lo que posibilita el aprendizaje desde y en múltiples direcciones.

En definitiva, la incorporación curricular de las prácticas de extensión como prácticas de Educación Experiencial implica para los estudiantes y docentes experimentar un proceso de aprendizaje 


\section{6}

el trabajo interdisciplinario, que compromete no solo la cooperación entre las disciplinas presentes en la formación sino, esencialmente, la construcción colectiva y consensual del proyecto de formación en situación de una intervención en el medio social con el abordaje de problemas concretos.

Lo relatado hasta aquí amerita nuevas reflexiones relacionadas con la evaluación de los procesos reflejados en las acciones que se vienen desarrollando. En este sentido, la Secretaría se encuentra trabajando con una meta que supone la evaluación institucional sobre las primeras experiencias implementadas de este tipo de prácticas. Coincidimos con Zulma Perassi (2011) cuando sostiene que la evaluación adquiere relevancia cuando emite un juicio de valor situado, es decir cuando muestra significados en un contexto social y político concreto.

Este número de la Revista $+E$, dedicado al tema "Integración de la docencia y la extensión. Un desafío para el currículo universitario", brinda la posibilidad de resignificar el sentido del aprendizaje experiencial y la lógica particular que posee esta práctica curricular en la UNL a partir de la difusión de algunas de estas experiencias relatadas por los propios protagonistas, en las cuales se aprecia el modo en que la incorporación de la extensión a los currículos de las carreras promueve experiencias de aprendizaje valiosas en la formación de los estudiantes y futuros graduados.

\section{Referencias bibliográficas}

Camilloni, A. (2013). La inclusión de la educación experiencial en el currículo universitario. En Menéndez, G. y otros, Integración docencia y extensión. Otra forma de aprender y de enseñar. Santa Fe: Ediciones UNL.

Dewey, J. (1960). Como pensamos. Nueva exposición de la relación ente el pensamiento reflexivo y proceso educativo. Barcelona: Paidós.

Menéndez, G. (2011). Los desafíos presentes y futuros de la extensión universitaria. Revista de Extensión Universitaria +E , (1), 22-31. Santa Fe: Ediciones UNL. Menéndez, G. (2012). Extensión y políticas públicas: la universidad en el centro del debate. Revista de Extensión Universitaria +E, (2), 40-46. Santa Fe: Ediciones UNL. Menéndez, G. (2013). La dimensión comunicacional de la extensión universitaria. En Menéndez, G. y otros, Integración docencia y extensión. Otra forma de aprender y enseñar (pp. 47-57). Santa Fe: Ediciones UNL.

Nicastro, S.; Greco, M. B. (2012). Entre trayectorias. Escenas y pensamientos en espacios de formación. $1^{\circ}$ Edición. $2^{\circ}$ reimpresión. Rosario: Homo Sapiens.

Perassi, Z. (2011). El desafío de evaluar un programa educativo. La experiencia de PROMSE en una provincia argentina ( $1^{\circ}$ ed.). Buenos Aires: Miño y Dávila. 